\title{
Vigilancia de la resistencia a antibióticos en enfermedad invasiva por Pseudomonas aeruginosa en Galicia: 2013-2014
}

\author{
Surveillance of antibiotics resistance producing invasive disease \\ for Pseudomonas aeruginosa in Galicia (Spain): 2013-2014
}

\author{
Lucía Martínez-Lamas ${ }^{1}$, Isabel Paz², Irene Rodríguez-Conde ${ }^{3}$, Francisco José Vasallo", \\ M. ${ }^{a}$ Ángeles Pallarés ${ }^{4}$, Fernando García-Garrote ${ }^{5}$, Isabel Losada ${ }^{6}$, Xurxo Hervada ${ }^{6}$
}

${ }^{1}$ Servicio de Microbiología. Complejo Hospitalario Universitario de Vigo. ${ }^{2}$ Servicio de Microbiología. Complejo Hospitalario Universitario de Ourense. ${ }^{3}$ Servicio de Microbiología. POVISA. Vigo. ${ }^{4}$ Servicio de Microbiología. Complejo Hospitalario Universitario de Pontevedra. ${ }^{5}$ Servicio de Microbiología. Hospital Universitario Lucus Augusti de Lugo. ${ }^{6}$ Dirección Xeral de Saúde Pública. Consellería de Sanidade. Xunta de Galicia. Grupo de trabajo para el estudio de la resistencias a antibióticos en Galicia.

\section{Resumen}

Introducción. El objetivo de este estudio es conocer la sensibilidad antibiótica de Pseudomonas aeruginosa productora de enfermedad invasiva en Galicia en 2013/2014, en el marco del Estudio de Vigilancia de las Resistencias Antimicrobianas.

Métodos. Se analizaron 357 aislamientos de $P$. aeruginosa en muestras de sangre o LCR en 9 hospitales de Galicia. Las variables fueron: procedencia, datos demográficos, tipo de muestra y sensibilidad antibiótica. Se usaron puntos de corte de CLSI. Para cada antibiótico se analizaron frecuencias, casos/100.000 habitantes, concordancia de la resistencia y diferencias entre hospitales, sexo y edad.

Resultados. El sexo predominante fue el masculino y la no sensibilidad superior en el grupo de 45 a 64 años con diferencias significativas a ciprofloxacino, imipenem, tobramicina y colistina. La no sensibilidad global: piperacilina/tazobactam $18 \%$, ciprofloxacino $28{ }^{\prime} 7 \%$, ceftazidima $17^{\prime} 1 \%$, cefepime $19^{\prime} 7 \%$, imipenem $23^{\prime} 1 \%$, meropenem $22{ }^{\prime} 1 \%$, tobramicina $13{ }^{\prime} 0 \%$; amikacina, 7'3\% y colistina 4'4\%. Los casos/100.000 habitantes fueron superiores en hombres según aumenta la edad. Sin analizar la colistina, el $57 ' 1 \%$ de los aislamientos fueron sensibles a los otros grupos estudiados (piperacilina/tazobactam, quinolonas, ceftazidima, aminoglucósidos, carbapenems), el 19'4\% fueron no sensibles a un antibiótico, 12'2\% a dos, el $3{ }^{\prime} 7 \%$ a tres, el $5^{\prime} 1 \%$ a cuatro y el $2{ }^{\prime} 0 \%$ a todos los analizados.

Conclusiones. De los antibióticos evaluados, los más activos frente a $P$. aeruginosa fueron amikacina y colistina. Nuestros datos concuerdan con lo observado a nivel nacional, excepto para colistina. Deben evaluarse periódicamente patrones de sensibilidad de $P$. aeruginosa en cada zona y cada hospital para poder valorar las diferentes pautas terapéuticas.

Palabras clave. Pseudomonas aeruginosa, no sensibilidad, estudio multicéntrico, Galicia.

\section{Introducción}

Pseudomonas aeruginosa es un reconocido patógeno nosocomial de distribución universal. Puede encontrarse en la piel, axilas, nasofaringe y perineo de individuos sanos, que son con frecuencia el origen de la infección nosocomial. Debido a su capacidad de colonización se acantona en el ambiente hospitalario en dispositivos, endoscopios, sistemas de ventilación mecánica y también en estancias y superficies de unidades de críticos y hemodiálisisis ${ }^{1,2}$. Es un microorganismo oportunista que habitualmente causa infecciones graves de elevada morbimortalidad en inmunodeprimidos oncohematológicos, en pacientes ingresados en unidades de

\begin{abstract}
Introduction. The aim of this study is to know the antibiotic sensitivity of the Pseudomonas aeruginosa, which produces invasive infections in Galicia in 2013/2014, in the framework of the Surveillance Study of Antimicrobial Resistance.

Methods. A total of 357 isolates of $P$. aeruginosa were analyzed in blood or CSF samples from 9 hospitals in Galicia. The variables were: origin, demographic data, sample type and antibiotic sensitivity. CLSI breakpoints were used. For each antibiotic we analyzed frequencies, cases/100.000 inhabitants, concordance of resistance and differences between hospitals, sex and age.

Results. The majority of patients were male gender and the not-sensitives were superior in the 45 to 64 age group with significant differences to ciprofloxacin, imipenem, tobramycin and colistin. The overall not-sensitivity isolates was: piperacillin/tazobactam $18 \%$, ciprofloxacin $28.7 \%$, ceftazidime $17.1 \%$, cefepime $19.7 \%$, imipenem $23.1 \%$, meropenem $22.1 \%$, tobramycin $13.0 \%$, amikacin $7.3 \%$ and colistin $4.4 \%$. The cases $/ 100,000$ inhabitants were higher in men as age increasing. Without analyzing colistin, the $57.1 \%$ of the isolates were sensitives to other antibiotics studied (piperacillin/tazobactam, quinolones, ceftazidime, aminoglycosides, carbapenems), $19.4 \%$ were not-susceptible to only one antibiotic, $12.2 \%$ to two, $3.7 \%$ to three, $5.1 \%$ to four and $2.0 \%$ to all antibiotics tested.

Conclusions. Of the antibiotics tested, the most susceptible to $P$. aeruginosa were amikacin and colistin. Our data are consistent with the observed ones nationwide except colistin. Sensitivity patterns of $P$. aeruginosa should be periodically evaluated in each area and each hospital in order to assess the different therapeutic regimens.

Key words. Pseudomonas aeruginosa, non-sensitivity, multicenter study, Galicia.
\end{abstract}

críticos, en tratamiento con antibióticos de amplio espectro, en grandes quemados y pacientes con fibrosis quística ${ }^{2}$. Las infecciones nosocomiales que más frecuentemente causa son neumonía, bacteriemia e infección de la herida quirúrgica y de la vía urinaria².

Pseudomonas aeruginosa es resistente a la mayoría de las penicilinas y cefalosporinas (excepto ceftazidima), tetraciclinas, cotrimoxazol y rifampicina. Esta resistencia se debe a la baja permeabilidad de la membrana externa, a la existencia de bombas activas de expulsión y a la producción de enzimas modificadoras, betalactamasas y carbapenemasas. Pseudomonas aeruginosa presenta una sensibilidad variable 
a piperacilina/tazobactam, ceftazidima, carbapenemas, aminoglucósidos y fluorquinolonas ${ }^{3}$.

El objetivo de este estudio es conocer la situación global de la sensibilidad antibiótica de Pseudomonas aeruginosa en infecciones invasivas (sangre y LCR) en la comunidad gallega. Para ello se llevó a cabo un análisis sobre la sensibilidad antibiótica de las cepas aisladas durante el bienio 2013-2014. Este estudio está en el marco del Estudio de resistencias a antimicrobianos en Galicia ${ }^{4,5}$ que se inició en 2007 con el estudio de no sensibilidades a tres microorganismos, que se amplió a 5 en 2013/2014, dentro de los cuales está la resistencia de Pseudomonas aeruginosa. Además, este estudio va encaminado a cumplir con las directrices que el Plan Nacional de Resistencias (PRAN) indica a las comunidades autónomas, y próximamente (2017) ampliará el estudio de vigilancia a 7 microorganismos.

\section{Material y métodos}

Se realizó un estudio retrospectivo en el que participaron 9 hospitales de Galicia. Esto comprende la casi totalidad de la población gallega, alrededor de 2.700.000 habitantes.

La fuente de información fueron los laboratorios de Microbiología de dichos hospitales, a los que se les solicitó el envío de los datos de sensibilidad de todas las cepas de Pseudomonas aeruginosa aisladas de sangre y líquido cefalorraquídeo, durante el bienio 2013 y 2014.

Recogida de datos. No se consideraron las cepas aisladas en un mismo paciente con menos de un mes de diferencia entre ellas.

Se solicitó a los centros participantes el envío al servicio de Epidemioloxía de la Dirección Xeral da Saúde Pública de una serie de datos referentes a la procedencia del paciente, datos demográficos (sexo, edad), tipo de muestra (sangre o LCR), fecha de recogida de la muestra, así como a los resultados de las CMI de los antibióticos analizados y su interpretación. Estos datos fueron introducidos en una base de datos común. Al final del estudio se realizó un informe con los resultados que fue validado por cada centro.

Estudio microbiológico. Los antibióticos testados fueron piperacilina/tazobactam, ciprofloxacino, cefotaxima, ceftazidima, cefepime, imipenem, meropenem, tobramicina, amikacina y colistina. La interpretación de los resultados se realizó siguiendo los criterios del CLSI.

Las pruebas de identificación bioquímica y de sensibilidad antibiótica fueron realizadas con los sistemas automatizados Microscan (Beckman, EEUU), Vitek 2 (Biomérieux, Francia) y BD Phoenix (Becton Dickinson, Bioscience), siguiendo la metodología de trabajo recomendada por los fabricantes, tanto para la identificación como para la lectura de los antibiogramas. La confirmación de algunas resistencias discrepantes se realizó por los métodos que contempla CLSI.

Estudio estadístico. Todos los datos proporcionados por los hospitales fueron registrados, almacenados y procesados en una base de datos con el programa Excel versión 2010. El análisis estadístico se realizó con la aplicación Epidat versiones 3.1 y 4.0 .
Se calcularon los porcentajes de no sensibilidad (sensibilidad intermedia y resistente) de $P$. aeruginosa con el intervalo de confianza del 95\% (IC95\%) a los antibióticos estudiados y se relacionaron con variables demográficas como la edad y sexo de los pacientes y también con los hospitales de procedencia de las cepas. Para determinar la asociación 0 independencia entre dos variables, se utilizó la prueba de Chi-cuadrado de Pearson y se consideró que había diferencias estadísticamente significativas cuando el valor de $p<0,05$. Para medir la concordancia de no sensibilidad entre dos antibióticos se utilizó el índice kappa, que se interpretó según los criterios de Altman'.

\section{Resultados}

Durante los años 2013 y 2014 se notificaron 357 aislamientos de P. aeruginosa, 181 en 2013 y 176 en 2014. Las muestras de las que procedieron los aislamientos fueron $17 \%$ de líquido cefalorraquídeo y 98 '3\% de sangre. El $66{ }^{\prime} 1 \%$ procedían de hombres y el 33 ' $9 \%$ de mujeres. Respecto a la edad, la no sensibilidad se incrementa en los mayores (tabla 1). La incidencia (casos/100.000 habitantes) de los aislamientos no sensibles de las infecciones invasoras por $P$. aeruginosa durante el período del estudio, por edad y sexo (tabla 2), de modo que fueron superiores en hombres que en mujeres y a medida que aumenta la edad, excepto para el grupo de 0 a 4 años.

Los resultados de la no sensibilidad en relación con los hospitales estudiados se muestran en la tabla 3.

Piperacilina/tazobactan. La no sensibilidad a piperacilina/ tazobactam ( $\mathrm{n}=300$ ) fue del 18'0\% (IC95\%, 13'5\%-22'5\%), con mayor no sensibilidad en POVISA 38' $5 \%$ ( $p=0$ '04) y menor en el CHUAC 9'4\% ( $\left.p=0^{\prime} 04\right)$ (tabla 3). No hubo diferencias por sexo 0 edad (tabla 1).

Ciprofloxacino. La no sensibilidad global a ciprofloxacino $(n=356)$ fue del $28^{\prime} 7 \%$ (IC95\%, 23'8\%-33'5\%) y no hubo diferencias significativas entre hombres y mujeres y aunque la prueba de tendencia lineal de la edad fue negativa, en el grupo de de 45 a 64 años el porcentaje de no sensibles fue estadísticamente significativo respecto al total (tabla 1).

Cefalosporinas. La no sensibilidad a ceftazidima $(n=357)$ fue del 17'1\%, (IC 95\% 13'04\%-21'1\%). No existió diferencia significativa respecto al sexo 0 la edad. En el CHOP, el alto porcentaje de aislamientos no sensibles fue estadísticamente significativo ( $p=0$ '002) en relación al resto de los hospitales (tabla 3).

La no sensibilidad global de $P$. aeruginosa a cefepime $(n=345)$ fue del 19'7\% (IC 95\% 15'4\%-24'05\%). No existieron diferencias significativas respecto al sexo 0 a la edad. Ese porcentaje fue más alto en el hospital en el CHOP y en el Arquitecto Marcide y en el HULA (tabla 3). Existe muy buena concordancia entre los aislamientos no sensibles a cefepime y ceftazidima, índice kappa=0'98 ( $\mathrm{p}<0^{\prime} 001$ ).

Carbapenems. Respecto a la no sensibilidad global a imipenem ( $n=347$ ) fue del 23'1\% (IC95\%, (IC95\%, 18'5\%$27^{\prime} 7 \%$ ). No hubo diferencias significativas en relación al 
Tabla 1. Porcentaje de no sensibilidad ( $\mathrm{n}=$ número de casos analizados) de $P$. aeruginosa por sexo y grupo de edad. Bienio 2013/2014.

\begin{tabular}{|c|c|c|c|c|c|c|c|c|c|c|c|}
\hline $2013 / 2014$ & $\begin{array}{l}\text { Piperacilina/ } \\
\text { tazobactam }\end{array}$ & \multicolumn{2}{|c|}{ ciprofloxacino } & \multicolumn{2}{|c|}{ ceftazidima } & cefepime & imipenem & meropenem & tobramicina & amikacina & colistina \\
\hline \multicolumn{12}{|l|}{ SEXO } \\
\hline $\begin{array}{l}\text { Hombre } \\
(n=236)\end{array}$ & $17^{\prime} 0(n=200)$ & \multicolumn{2}{|c|}{$26^{\prime} 0(n=235)$} & \multicolumn{2}{|c|}{$16^{\prime} 9(n=236)$} & $21^{\prime} 6(n=227)$ & $23^{\prime} 1(n=229)$ & $21^{\prime} 8(n=220)$ & $10^{\prime} 4(n=230)$ & $4^{\prime} 7(n=211)$ & $4^{\prime} 7(n=193)$ \\
\hline $\begin{array}{l}\text { Mujer } \\
(n=121)\end{array}$ & $20^{\prime} 0(n=100)$ & \multicolumn{2}{|c|}{$33^{\prime} 9(n=121)$} & \multicolumn{2}{|c|}{$17^{\prime} 4(n=121)$} & $16^{\prime} 1(n=118)$ & $22^{\prime} 9(n=118)$ & $22^{\prime} 7(n=110)$ & $18^{\prime} 3(n=115)$ & $\begin{array}{c}12^{\prime} 4(n=105) \\
\left(p=0^{\prime} 01\right)\end{array}$ & $4^{\prime} 0(n=100)$ \\
\hline TOTAL & $18^{\prime} 0(n=300)$ & \multicolumn{2}{|c|}{$28^{\prime} 7(n=355)$} & \multicolumn{2}{|c|}{$17^{\prime} 1(n=357)$} & $19^{\prime} 7(n=345)$ & $23^{\prime} 1(n=347)$ & $22 ' 1(n=330)$ & $13^{\prime} 0(n=345)$ & $7^{\prime} 3(n=316)$ & $4^{\prime} 4(n=293)$ \\
\hline 2013/2014 & \multicolumn{2}{|c|}{$\begin{array}{l}\text { Piperacilina/ } \\
\text { tazobactam }\end{array}$} & \multicolumn{2}{|c|}{ ciprofloxacino } & ceftazidima & cefepime & imipenem & meropenem & tobramicina & amikacina & colistina \\
\hline \multicolumn{12}{|c|}{ GRUPO EDAD (AÑOS) } \\
\hline 0 a $4(n=2)$ & \multicolumn{2}{|c|}{$50(n=2)$} & $50^{\prime} 0(n=2$ & & $50^{\prime} 0(n=2)$ & $50^{\prime} 0(\mathrm{n}=2)$ & $50^{\prime} 0(\mathrm{n}=2)$ & $50^{\prime} 0(\mathrm{n}=2)$ & $50^{\prime} 0(\mathrm{n}=2)$ & $100(n=1)$ & $0^{\prime} 0(\mathrm{n}=2)$ \\
\hline 5 a $14(n=1)$ & $0^{\prime} 0(n=1$ & & $0^{\prime} 0(n=1)$ & & $0^{\prime} 0(n=1)$ & $0^{\prime} 0(n=1)$ & $0^{\prime} 0(n=1)$ & $0^{\prime} 0(n=1)$ & $0(n=1)$ & $0^{\prime} 0(\mathrm{n}=1)$ & $0^{\prime} 0(n=1)$ \\
\hline 15 a $44(n=2$ & $15^{\prime} 0(n=2$ & & $28^{\prime} 6(n=2$ & & $24{ }^{\prime} 1(n=29)$ & $24{ }^{\prime} 1(n=29)$ & $35^{\prime} 7(n=28)$ & $33^{\prime} 3(n=27)$ & $18^{\prime} 5(n=27)$ & $0^{\prime} 0(n=27)$ & $0^{\prime} 0(n=24)$ \\
\hline 45 a $64(n=7$ & $24^{\prime} 2(n=6$ & & $\begin{array}{r}44^{\prime} 6(n=7 \\
\left(p<00^{\prime} 001\right.\end{array}$ & & $21^{\prime} 6(n=74)$ & $23^{\prime} 9(n=71)$ & $\begin{array}{c}39^{\prime} 7(n=73) \\
\left(p<0^{\prime} 001\right)\end{array}$ & $37 ' 1(n=70)$ & $\begin{array}{c}23^{\prime} 9(\mathrm{n}=71) \\
\left(\mathrm{p}=0^{\prime} 002\right)\end{array}$ & $9^{\prime} 7(n=62)$ & $\begin{array}{c}9^{\prime} 5(n=63) \\
\left(p=0^{\prime} 02\right)\end{array}$ \\
\hline 65 a $74(n=9$ & $15^{\prime} 9(n=8$ & & $21^{\prime} 9(n=9$ & & $17^{\prime} 7(n=96)$ & $17^{\prime} 8(n=90)$ & $16^{\prime} 0(n=94)$ & $13^{\prime} 5(n=89)$ & $9^{\prime} 7(\mathrm{n}=93)$ & $11^{\prime} 6(n=86)$ & $3^{\prime} 9(n=77)$ \\
\hline$\geq 75(n=154)$ & $16^{\prime} 5(n=13$ & & $25^{\prime} 3(n=15$ & & $13(n=154)$ & $17^{\prime} 8(n=152)$ & $16^{\prime} 9(n=148)$ & $17^{\prime} 19(n=140)$ & $8^{\prime} 7(n=150)$ & $4^{\prime} 3(n=139)$ & $3^{\prime} 2(n=126)$ \\
\hline TOTAL & $18^{\prime} 0(n=3 c$ & 300) & $28^{\prime} 7(n=35$ & & $17^{\prime} 1(n=356)$ & $19^{\prime} 7(n=345)$ & $23^{\prime} 1(n=346)$ & $22^{\prime} 1(n=329)$ & $13^{\prime} 0(n=344)$ & $7^{\prime} 3(n=316)$ & $4^{\prime} 4(n=293)$ \\
\hline
\end{tabular}

sexo. Según la edad, la no sensibilidad en el grupo de 45 a 64 años fue superior en relación al resto, y esa diferencia fue estadísticamente significativa (tabla 2).

Se estudió la no sensibilidad a meropenem $(n=329)$ y fue del 22 '1\% (IC95\%, 17'5\%-26'9\%). No se encontraron diferencias significativas respecto al sexo. Como en el otro carbapenem estudiado, imipenem, la no sensibilidad fue superior en el grupo de 45 a 64 años (tabla 1). La concordancia de la no sensibilidad de imipenem y meropenem fue buena, índice kappa $=0$ '74 ( $p<0$ '001). También se calculó la concordancia entre la no sensibilidad entre imipenem y ciprofloxacino, dando como resultado una concordancia débil (Kappa=0'36).

Aminoglucósidos. El 13'0\% (IC 95\%, 9'3\%-16'7\%) de los aislamientos $(n=345)$ fueron no sensibles a tobramicina con diferencias estadísticamente significativas entre hombres y mujeres (tabla 1). En el grupo de 45 a 64 Años hubo un porcentaje de aislamientos no sensibles mayor que el global con diferencia estadísticamente significativa en relación al resto de los grupos de edad (tabla 1). Según los hospitales destaca el alto porcentaje de cepas no sensibles en el CHOP $43{ }^{\prime} 9 \%$ ( $\mathrm{p}<0$ '001), que supone el doble del porcentaje de resistencia global (tabla 3 ).

En relación a la amikacina ( $n=316)$ el 7'3\% (IC95\%, 4'25\%10 '3\%) fue resistente. Fue más frecuente en hombres $\left(p=0^{\prime} 01\right)$. No hubo diferencias según el hospital de procedencia de los aislamientos.

Colistina. El porcentaje de aislamientos no sensibles a colistina fue del 4'4\% (IC 95\% 1'9\%-6'7\%). Solo hubo dos aislamientos clasificados como resistentes, ambos en el CHUO. Hubo 11 aislamientos con sensibilidad intermedia, 4 de ellos en el CHUAC, con diferencia significativa en relación al resto $(p=0$ '01). No hubo diferencias en relación al sexo, pero sí en cuanto a la edad (tabla 1).

Tabla 2. Casos/100.000 habitantes de las infecciones por P. aeruginosa 2013 a 2014.

\begin{tabular}{|c|c|c|c|c|c|c|c|c|c|}
\hline $2013 / 2014$ & $\begin{array}{l}\text { Piperacilina/ } \\
\text { tazobactam }\end{array}$ & ciprofloxacino & ceftazidima & cefepime & imipenem & meropenem & tobramicina & amikacina & colistina \\
\hline 0 a 4 & $0{ }^{\prime} 9$ & 0 '9 & 0 '9 & 0 '9 & 0 '9 & 0 '9 & 0 '9 & $0 ' 9$ & $0^{\prime} 0$ \\
\hline 5 a 14 & $0^{\prime} 0$ & $0^{\prime} 0$ & $0^{\prime} 0$ & 0 '0 & 0 '0 & 0 '0 & 0'0 & 0 '0 & 0 '0 \\
\hline 15 a 44 & 0,3 & $0{ }^{\prime} 8$ & 07 & $0{ }^{\prime} 7$ & $1^{\prime} 0$ & $0{ }^{\prime} 9$ & 0 '5 & 0 '0 & $0^{\prime} 0$ \\
\hline 45 a 64 & $2^{\prime} 0$ & $4^{\prime} 3$ & $2 ' 1$ & $2^{\prime} 2$ & $3 ' 9$ & $3^{\prime} 4$ & $2 ' 2$ & 0'8 & 0'8 \\
\hline 65 a 74 & $4^{\prime} 3$ & $7^{\prime} 0$ & 57 & $5^{\prime} 3$ & $5{ }^{\prime} 0$ & $4^{\prime} 0$ & $3{ }^{\prime} 0$ & $3{ }^{\prime} 3$ & $1^{\prime} 0$ \\
\hline$\geq 75$ & $6^{\prime} 3$ & $11 ' 1$ & 57 & $7^{\prime} 8$ & $7 ' 1$ & $7 ' 1$ & 37 & $1^{\prime} 7$ & $1 ' 1$ \\
\hline Hombre & $2^{\prime} 6$ & $4^{\prime} 6$ & $3{ }^{\prime} 0$ & $3 ’ 7$ & $4^{\prime} 0$ & $3{ }^{\prime} 6$ & $1 ' 8$ & $0 ’ 75$ & $0 ’ 7$ \\
\hline Mujer & $1 ' 4$ & $2^{\prime} 9$ & $1^{\prime} 5$ & $1^{\prime} 3$ & $1^{\prime} 9$ & $1 ' 8$ & $1^{\prime} 5$ & 0 '9 & $0 ' 3$ \\
\hline
\end{tabular}


Tabla 3. Porcentaje de no sensibilidad ( $n=$ número de casos analizados) de $P$. aeruginosa por hospital. Bienio 2013/2014.

\begin{tabular}{|c|c|c|c|c|c|c|c|c|c|c|}
\hline 2013/2014 & $\begin{array}{c}\text { Piperacilina/ } \\
\text { tazobactam } \\
(n=300)\end{array}$ & $\begin{array}{c}\text { Ciprofloxacino } \\
(n=356)\end{array}$ & $\begin{array}{c}\text { Ceftazidima } \\
(n=357)\end{array}$ & $\begin{array}{c}\text { Cefepime } \\
(n=345)\end{array}$ & $\begin{array}{c}\text { Imipenem } \\
(n=347)\end{array}$ & $\begin{array}{c}\text { Meropenem } \\
(n=330)\end{array}$ & $\begin{array}{c}\text { Tobramicina } \\
(n=345)\end{array}$ & $\begin{array}{c}\text { Amikacina } \\
(n=316)\end{array}$ & $\begin{array}{c}\text { Colistina } \\
(n=293)\end{array}$ \\
\hline HOSPITAL
\end{tabular}

Test estadístico: chi-cuadrado de Pearson: *'ignificación estadística $p<0$ '05 ${ }^{\star *}$ cepas con sensibilidad intermedia

Multirresistencia. Comparación EARS-N. Se analizó la multirresistencia de $P$. aeruginosa a antibióticos seleccionados según el criterio del EARS-N. En el 82'4\% de los aislamientos constan los datos de la no sensibilidad a los 5 antibióticos analizados por el EARS-N. Los resultados obtenidos se compararon con los del EARS-N del año 2013 (tabla 4).

\section{Discusión}

En este trabajo se presentan por primera vez los datos para toda Galicia sobre el nivel de no sensibilidad de los aislamientos de $P$. aeruginosa frente a un grupo de antimicrobianos activos para este microorganismo. Este estudio multicéntrico muestra resultados representativos de toda la Comunidad Autónoma. El escaso número de aislamientos en relación con el alto número de hospitales participantes puede ser atribuido porque solo se estudiaron muestras invasivas de sangre y LCR, y estas infecciones suelen ser más frecuentes a nivel respiratorio 0 urinario.

$P$. aeruginosa es intrínsecamente resistente a un gran número de antimicrobianos. Así mismo, la resistencia a los antimicrobianos anti-pseudomónicos es común en Europa con porcentajes de resistencia mayores del $10 \%$ para la mayoría de ellos, excepto en el grupo de las polimixinas que todavía mantienen un $2 \%$ de resistencia. En los últimos años se ha puesto de manifiesto un incremento significativo en la no sensibilidad a carbapenems en España y a nivel europeo, junto con un descenso de la misma en el grupo de fluoroquinolonas y aminoglucósidos (4\%)

De los antibióticos evaluados en el estudio, los más activos frente a $P$. aeruginosa fueron amikacina y colistina, cuya resistencia se objetivó por debajo del 10\%. La resistencia a ceftazidima y piperacilina/tazobactam aunque superior al $10 \%$ todavía mantiene porcentajes aceptables para el tra- tamiento empírico de las infecciones por este tipo de microorganismo, a pesar haber sido antibióticos ampliamente utilizados para su tratamiento. En nuestra área se constata un elevado porcentaje de resistencia a carbapenems con un valor cercano al 23\%, con bajos porcentajes de resistencia a aminoglucósidos (tobramicina 13’0\%, 7’3\% colistina), probablemente relacionados con las precauciones en su uso a fin de reducir la toxicidad renal ${ }^{8}$. La resistencia a quinolonas es elevada con porcentajes cercanos al 30\%, lo que corrobora el posible ascenso de la misma y posterior estabilización en nuestro país durante los últimos años, probablemente sea reflejo del uso extendido a nivel comunitario9,10. En general, la exposición a cualquier anti-pseudomónico se relaciona con la inducción de resistencia, aunque este efecto es mayor en fluoroquinolonas y carbapenems ${ }^{11,12,13}$, lo que concuerda con los elevados porcentajes de resistencia obtenidos para ambos en nuestra comunidad autónoma.

Casal et al/4, en un estudio que reportaba la evolución de las resistencia de $P$. aeruginosa en España con aislamientos procedentes de muestras principalmente no invasivas, en el período 2005 a 2010, indicaba tendencia de la resistencia estable, con menor resistencia en tobramicina y meropenem y mayor en gentamicina, ciprofloxacino y cefepima. Estudios recientes, muestran tasas de resistencia a uno o dos antimicrobianos que alcanzan el $24 \%$ con porcentajes de multirresistencia y resistencia extrema de $33 \%$ y $10 \%$, respectivamente. No obstante las tasas de multirresistencia (resistencia a tres 0 más grupos de antimicrobianos) y resistencia extrema son inferiores en nuestro estudio que las observadas a nivel nacional ${ }^{15}$. Sin embargo se encuentran cercanas al nivel europeo $(12,4 \%)^{7}$. En nuestra comunidad, en comparación con los informes de la Red de Vigilancia Europea (EARS-N 2013) el porcentaje de sensibilidad a todos los fármacos incluidos en el estudio fue 


\section{Sensibilidad combinada de P. aeruginosa a diferentes antibióticos y su comparación con el EARS-N 2013}

\begin{tabular}{|c|c|c|c|}
\hline \multirow{7}{*}{ no sensibilidad única } & & & 2013 \\
\hline & & 19,4 & 13,6 \\
\hline & ceftazidima & 0 & 0,8 \\
\hline & quinolonas & 10,5 & 4,1 \\
\hline & carbapenemes & 6,1 & 4,9 \\
\hline & Piperacilina/tazobactam & 1 & 1,6 \\
\hline & aminoglucósidos & 1,7 & 2,2 \\
\hline \multirow[t]{11}{*}{ no sensibilidad doble } & & 12,2 & 7,6 \\
\hline & quinolonas+aminoglucósidos & 2,4 & 2,2 \\
\hline & Piperacilina/tazobactam+ceftazidima & 4,8 & $20^{\prime}$ \\
\hline & quinolonas+carbapenemes & 3,4 & 1,3 \\
\hline & aminoglucósidos+carbapenemes & 0,3 & 0,5 \\
\hline & Piperacilina/tazobactam+carbapenemes & 1 & 0,5 \\
\hline & Piperacilina/tazobactam+quinolonas & 0 & 0,3 \\
\hline & ceftazidima+carbapenemes & 0,3 & 0,2 \\
\hline & quinolonas+ceftazidima & 0,3 & 0,2 \\
\hline & Piperacilina/tazobactam+aminoglucosidos & 0 & 0,2 \\
\hline & ceftazidima+aminoglucosidos & 0 & 0,1 \\
\hline \multirow[t]{11}{*}{ no sensibilidad triple } & & 3,7 & 5,4 \\
\hline & quinolonas+aminoglucósidos+carbapenemes & 0,3 & 1,8 \\
\hline & Piperacilina/tazobactam+ceftazidima+carbapenemes & 2,4 & 0,9 \\
\hline & Piperacilina/tazobactam+quinolonas+aminoglucósidos & 0 & 0,8 \\
\hline & Piperacilina/tazobactam+quinolonas+ceftazidima & 0,3 & 0,5 \\
\hline & Piperacilina/tazobactam+quinolonas+carbapenemes & 0,7 & 0,3 \\
\hline & quinolonas+ceftazidima+aminoglucosidos & 0 & 0,3 \\
\hline & quinolonas+ceftazidima+carbapenemes & 0 & 0,3 \\
\hline & piperacilina+carbapenemes+aminoglucosidos & 0 & 0,2 \\
\hline & Piperacilina/tazobactam+ceftazidima+aminoglucosidos & 0 & 0,2 \\
\hline & ceftazidima+aminoglucosidos+carbapenemes & 0 & 0,1 \\
\hline \multirow[t]{5}{*}{ no sensibilidad cuadruple } & & 5,1 & 4,3 \\
\hline & Piperacilina/tazobactam+quinolonas+aminoglucósidos+carbap & 0,7 & 1,3 \\
\hline & quinolonas+ceftazidima+aminoglucósidos+carbapenemes & 0,3 & 1,2 \\
\hline & ceftazidima+quinolonas+piperacilina/tazobactam+aminoglucósi & 1,4 & 0,9 \\
\hline & Piperacilina/tazobactam+fluorquinolonas+ceftazidima+carbape & 2,7 & 0,7 \\
\hline resistentes a todos & & 2,0 & 4,6 \\
\hline
\end{tabular}

inferior ( $57,1 \%$ vs. $64,5 \%$ ), la panresistencia (resistencia a todos los fármacos excepto a la colistina) inferior en casi un $2 \%$, al igual que la no sensibilidad triple. No obstante, la no sensibilidad única, doble y cuádruple superó a la obtenida en el estudio europeo, siendo el incremento especialmente relevante en el caso de la no sensibilidad única y doble.

A pesar de la correlación observada entre los aislamientos no sensibles a ceftazidima y cefepime, así como la buena concordancia entre los carbapenems estudiados, la inferencia de los resultados no es posible dado que en ambos casos pueden generarse fenotipos de resistencia selectivos frente a alguno de ellos ${ }^{16,17}$.

Dado el creciente problema derivado de la multirresistencia en $P$. aeruginosa, se ha incrementado el uso de colistina para el tratamiento de las infecciones causadas por estas cepas. Las causas de la resistencia a colistina todavía no están totalmente aclaradas y en su mayoría son el resultado de mecanismos adaptativos y por tanto reversibles con la suspensión del tratamiento ${ }^{18}$.
Sorprendentemente, en nuestra comunidad la tasa de no sensibilidad a colistina es del 4,4\%, siendo una cifra elevada en comparación con los niveles europeos $(2 \%)^{7}$, y con los resultados del estudio de Cagot et al ${ }^{15}$, que como nosotros estudia las resistencias de P. aeruginosa en muestras de sangre, y que reportan una resistencia a colistina del 3\% utilizando los puntos de corte con los criterios de EUCAST. En nuestro estudio tan sólo dos aislamientos se consideraron resistentes, los otros tuvieron sensibilidad intermedia resaltando que las pruebas de sensibilidad a colistina sólo se evaluaron en 293 de 357 aislamientos estudiados.

Este estudio presenta las limitaciones de los estudios retrospectivos, con mayor riesgo de sesgos de selección e información. Además, no ha sido posible diferenciar si los aislados correspondían a infecciones nosocomiales o fueron adquiridos previamente al ingreso del paciente. En general, como se ha demostrado en estudios previos los aislamientos de $P$. aeruginosa nosocomiales son más resistentes a antibióticos anti-pseudomónicos, que los comunitarios, aunque 
Ios niveles de resistencia en los aislamientos comunitarios son también destacables ${ }^{19}$. Por otro lado no se dispone de datos sobre los mecanismos moleculares implicados en la resistencia de nuestros aislamientos.

El análisis de los factores de riesgo para la aparición de multirresistencia en $P$. aeruginosa es muy complejo, dada la diversidad de mecanismos implicados y la variabilidad de las bases genéticas que los sustentan, asociándose la presencia de Pseudomonas multirresistente a un tipo de paciente 0 enfermedades concretas (fibrosis quística, institucionalización entre otras) ${ }^{20}$. Probablemente, esta sea la causa del incremento de resistencia con la edad observado en nuestros aislamientos, ya que son los pacientes de edad avanzada los que más frecuentemente presentan alguno de estos factores de riesgo y esto está en relación con el espectro de población de nuestra comunidad en la cual la población tiende al envejecimiento. En nuestro caso, las diferencias encontradas entre los distintos hospitales se pueden explicar por el distinto uso de los antibióticos en cada centro, por las variaciones geográficas de los mecanismos de resistencia de $P$. aeruginosa, las diferencias que existen entre los hospitales en relación con la edad y características demográficas de la población atendida, así como de los tipos de procesos asistenciales y pacientes ingresados en cada uno de los hospitales. El elevado porcentaje de resistencias presentado en el CHOP en relación al resto de los hospitales es debido a un brote que se produjo a lo largo del año 2014 y al escaso tamaño muestral.

Estas diferencias avalan la importancia de realizar estudios de los patrones de sensibilidad de $P$. aeruginosa en cada zona, incluso de cada hospital en particular, y hacerlo periódicamente, para poder valorar las diferentes pautas terapéuticas posibles. Esto está en el marco que promulga el PRAN, y a partir de estos resultados se podrían realizar guías clínicas para orientar sobre el manejo de este tipo de infecciones. Es muy importante vigilar la resistencia a colistina, dado a la elevada tasa de no sensibilidad en nuestra área, en relación con otros estudios publicados.

\section{Bibliografía}

1. Blondel-Hill E, Henry DA, Speert DP. Pseudomonas. En: Murray PR, Baron EJ, Jorgensen JH, Landry ML, Pfaller MA. Manual of Clinical Microbiology. 9th Edition, ASM Press, Washington DC. 2007; p. 734-748.

2. D'Agata E. Pseudomonas aeruginosa and other Pseudomonas species. En: Mandell, Douglas and Bennett's. Principles and Practice of Infectious Diseases. 8th Edition, Churchill-Livingstone. ELSEVIER. 2015; p. 2518-2531.

3. Vila J, Marco F. Lectura interpretrada del antibiograma de bacilos gramnegativos no fermentadores. Enferm. Infecc. Microbiol Clin 2010; 28(10):426-436.
4. Treviño M, Losada I, Pallarés MA, Vasallo FJ, Coira A, Fernández-Pérez B et al. Vigilancia de la resistencia a los antibióticos en Staphylococcus aureus en Galicia: 2007-2012 Rev Esp Quimioter 2015; 28(6): 289-294.

5. Treviño M, Losada I, Fernández-Pérez B, Coira A, Peña-Rodríguez MF, Hervada X et al. Vigilancia de la sensibilidad a antimicrobianos de Escherichia coli productor de infecciones del tracto urinario comunitarias en Galicia. Rev Esp Quimioter 2016; 29(2): 86-90.

6. Altman DG. Practical Statistics for Medical Research. New York. Chapman and Hall, 1991.

7. The European Centre for Disease Prevention and Control. Antimicrobial resistance surveillance in Europe. Annual report of the European Antimicrobial Resistance Surveillance Network (EARS-Net)—2014. Available at: http://ecdc.europa.eu/en/publications/Publications/antimicrobial-resistance-europe-2014.pdf.

8. Asencio MA, Huertas M, Carranza R, Franco M, Castellanos J, Barberá JR et al. Tendencia de sensibilidad de los patógenos bacterianos más frecuentemente aislados en el Hospital General La Mancha Centro durante el periodo 2010-2012. Rev Esp Quimioter 2014; 27(4): 261-8.

9. Sanchez-Romero I, Cercenado E, Cuevas 0, García-Escribano N, García-Martínez J, Bouza E et al. Evolution of the antimicrobial resistance of Pseudomonas aeruginosa in Spain: second national study (2003). Rev Esp Quimioter 2007; 20(2): 222-9.

10. Gasink LB, Fishman NO, Weiner MG, Nachamkin I, Bilker WB, Lautenbach E. Fluoroquinolone-Resistant Pseudomonas aeruginosa: Assessment of Risk Factors and Clinical Impact. Am J Med 2006; 119(6):526.e19-25.

11. Riou M, Avrain L, Carbonnelle S, El Garch F, Pirnay J-P, De Vos D et al. Increase of efflux-mediated resistance in Pseudomonas aeruginosa during antibiotic treatment in patients suffering from nosocomial pneumonia. Int J Antimicrob Agents 2016; 47(1): 77-83.

12. Livermore DM. Of Pseudomonas, porins, pumps and carbapenems. J Antimicrob Chemother 2001; 47(3): 247-50.

13. Livermore DM. "Multiple mechanisms of antimicrobial resistance in Pseudomonas aeruginosa: our worst nightmare?" Clin Infect Dis 2002; 34(5): 634-40.

14. Casal MM, Causse M, Rodríguez-López F, Casal M. Resistencia antimicrobiana en aislados clínicos de P. aeruginosa. Rev Esp Quimioter 2012; 25(1): 37-41

15. Cabot G, Ocampo-Sosa AA, Tubau F, Macia MD, Rodríguez C, Moya B et al. Overexpression of $\mathrm{AmpC}$ and efflux pumps in Pseudomonas aeruginosa isolates from bloodstream infections: prevalence and impact on resistance in a Spanish multicenter study. Antimicrob Agents Chemother 2011; 55(5): 906-11.

16. Pragasan Ak, Raghanivedha M, Anandan S, Veeraraghavan B. Characterization of Pseudomonas aeruginosa with discrepant carbapenem susceptibility profile. Ann Clin Microbiol Antimicrob 2016; 15: 12

17. Peña C, Suárez C, Tubau F, Juan C, Moya B, Domínguez MA et al. Nosocomial outbreak of a non-cefepime-susceptible ceftazidime-susceptible Pseudomonas aeruginosa strain overexpressing MexXY-OprM and producing an integron-borne PSE-1 betta-lactamase. J Clin Microbiol 2009; 47(8): 2381-7.

18. Lee JY, Park YK, Chung ES, Na IY, Ko KS., Evolved resistance to colistin and its loss due to genetic reversion in Pseudomonas aeruginosa. Sci Rep 2016; 6: 25543.

19. McGregor JC, Bearden DT, Townes JM, Sharp SE, Gorman PN, Elman MR et al. Comparison of antibiograms developed for inpatients and primary care outpatients. Diagn Microbiol Infect Dis 2013; 76(1): 73-9.

20. Aloush V, Navon-Venezia S, Seigman-Igra Y, Cabili S, Carmeli Y. Multidrug-resistant Pseudomonas aeruginosa: risk factors and clinical impact. Antimicrob Agents Chemother 2006; 50(1): 43-8.

\section{Grupo de trabajo de estudio de resistencias a antibioticos en Galicia}

Gema Barbeito. Servicio de Microbiología. Complejo Hospitalario Universitario de Santiago de Compostela.

M. ${ }^{\text {a }}$ Fernanda Peña. Servicio de Microbiología. Hospital Arquitecto Marcide de Ferrol.

M. ${ }^{\text {a }}$ Begoña Fernández-Pérez. Servicio de Microbiología. Complejo Hospitalario Universitario de A Coruña.

Gael Naveira. Dirección Xeral de Saúde Pública. Consellería de Sanidade. Xunta de Galicia.

Elisabeth Prieto. Servicio de Microbiología. Hospital da Costa. Burela.

Agradecimientos. Nuestro agradecimiento a la Sociedad Gallega de Microbiología (SOGAMIC) y a la Dirección Xeral de Saúde Pública (DXSP) por respaldar este proyecto.

Conflicto de intereses. Todos los autores declaran no tener conflicto de intereses. 